\title{
ENFERMEDADES INFECCIOSAS RELACIONADAS CON EL AGUA EN EL PERÚ
}

\author{
César Cabezas Sánchez 1 ,2,a
}

\begin{abstract}
RESUMEN
El crecimiento de la población mundial ha incrementado la demanda de agua, por otro lado, el cambio climático nos muestra que las fuentes de agua que antes eran seguras actualmente ya no lo son. Existen desigualdades entre las zonas urbanas y rurales, así el $96 \%$ de la población mundial urbana utiliza fuentes de agua potable frente al $84 \%$ de la población rural, mientras que el $82 \%$ de la población urbana frente al $51 \%$ de la población rural utiliza instalaciones de saneamiento. En el Perú el $80,4 \%$ de viviendas se provee de agua por red pública. En el área urbana, este servicio cubre $83,2 \%$; mientras que en el área rural el $71,3 \%$ de las viviendas cuenta con servicio higiénico conectado a red pública. Todo lo anterior condiciona la presencia de enfermedades infecciosas relacionadas con el agua como las diarreas, malaria, dengue, leptospirosis, hepatitis virales A y E, cuya clasificación, se muestra en este artículo. En el mundo las diarreas son la tercera causa de muerte entre menores de cinco años y más de 340000 niños menores de cinco años mueren por enfermedades diarreicas. Una de las metas de los Objetivos de Desarrollo Sostenible (ODS) es la relacionada al acceso universal al agua potable, saneamiento e higiene, lo que no puede lograrse sin prestar la debida atención a las desigualdades en el acceso observadas entre diferentes grupos, evitando las consecuencias de estas deficiencias tanto en nuestro país como en el mundo.
\end{abstract}

Palabras clave: Agua; Enfermedades transmisibles; Perú (fuente: DeCS BIREME).

\section{INFECTIOUS DISEASES RELATED TO WATER IN PERU}

\begin{abstract}
The growth of the world population has increased the demand for water. On the other hand, climate change shows us that the water sources that were previously safe are not anymore. There are inequalities between urban and rural areas, thus $96 \%$ of the urban world population uses drinking water sources compared to $84 \%$ of the rural population, while $82 \%$ of the urban population versus $51 \%$ of the rural population use sanitation facilities. In Peru, $80.4 \%$ of homes are supplied with water through a public network. In the urban area, this service covers $83.2 \%$; while in the rural area $71.3 \%$ of homes have a toilet service connected to the public network. All the above conditions the presence of water-related infectious diseases, such as diarrhea, malaria, dengue, leptospirosis, viral hepatitis A, and E, whose classification is shown in this article. In the world, diarrhea is the third cause of death among children under five years of age and more than 340,000 children under the age of five die from diarrheal diseases due to poor sanitation. One of the goals of the SDGs is related to universal access drinking water, sanitation and hygiene, which cannot be achieved without due attention to the inequalities in access observed between different groups, avoiding the consequences of these deficiencies both in our country and in the world.
\end{abstract}

Keywords: Water; Communicable diseases; Peru (source: MeSH NLM).

\section{INTRODUCCIÓN}

Sin agua es poco probable la vida y es indefectiblemente esencial para la salud humana, la producción de alimentos y el saneamiento; además de otros usos en la actividad humana, por lo que todos debemos ser conscientes de los recursos hídricos actuales, futuros y de las estrategias que se deben tener para una gestión eficaz que nos permita tener acceso a ella.

El crecimiento de la población humana y por consiguiente el incremento de la demanda de agua; así como, el cambio

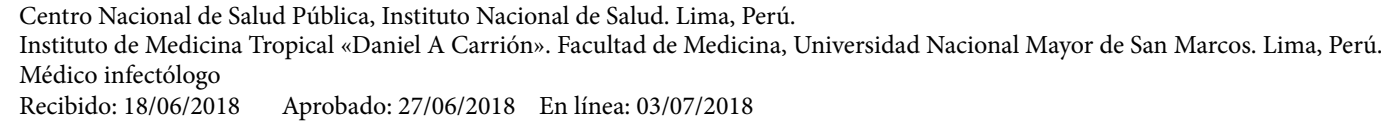

Citar como: Cabezas C. Enfermedades infecciosas relacionadas con el agua en el Perú. Rev Peru Med Exp Salud Publica. 2018;35(2):309-16. doi: 10.17843/ rpmesp.2018.352.3761. 
climático nos muestra que las fuentes de agua que antes eran seguras actualmente ya no lo sean y está en riesgo su disponibilidad en el futuro. De otro lado, los proyectos en materia de agua con frecuencia son extensos y costosos, y culminarlos toma varios años, por lo que la planificación futura es crucial ${ }^{(1,2)}$.

El crecimiento de la poblacional mundial y los avances respecto a la disponibilidad de agua potable, instalaciones de saneamiento y la disposición de excretas al aire libre se pueden apreciar en la Tabla 1, una tendencia que podría seguir mejorando.

Asimismo, hay cada vez más evidencias que el cambio climático puede alterar la incidencia de enfermedades transmitidas por el agua, y las enfermedades diarreicas en particular. Hay información histórica de la relación entre incidencia del clima y la diarrea. En las consideraciones de las relaciones históricas, la integración de estos componentes ayuda a identificar poblaciones vulnerables y priorizar estrategias de adaptación ${ }^{(3-5)}$. Igualmente es evidente que el fenómeno de El Niño incrementa el riesgo y aparición de enfermedades asociadas con el agua, lo cual se ha hecho evidente en diferentes periodos en el Perú (6).

Cuando se toca el tema del agua es importante considerar los determinantes para su acceso por parte de la población y considerar que existen desigualdades entre las zonas urbanas y rurales. Por ejemplo, el $96 \%$ de la población mundial urbana utiliza fuentes de agua potable mejoradas, frente al $84 \%$ de la población rural; mientras que, el $82 \%$ de la población mundial urbana frente al $51 \%$ de la población rural utiliza instalaciones de saneamiento mejoradas. Si se sigue con la velocidad actual de reducción de estas desigualdades, la práctica de la defecación al aire libre no se eliminará entre las poblaciones más pobres de las zonas rurales para el año $2030^{(2)}$.

Una de las enfermedades que más se relaciona con el agua insegura es la enfermedad diarreica que constituye la tercera causa de muerte entre menores de cinco años en el mundo ${ }^{(1)}$. Se calcula que más de 340000 niños menores de cinco años mueren anualmente por enfermedades diarreicas debidas a un saneamiento deficiente, siendo casi 1000 niños al día. Unos 161 millones de niños sufren retraso del crecimiento o malnutrición crónica, lo que está vinculado con la falta de agua, saneamiento e higiene, y en particular con la defecación al aire libre. Cada año podrían evitarse 842000 muertes con la mejora del agua, el saneamiento y la higiene; en ese contexto las deficiencias de estas condiciones contribuyen en gran medida a las enfermedades tropicales desatendidas ${ }^{(1)}$.

Frente a esta situación, líderes mundiales adoptaron un conjunto de objetivos globales para erradicar la pobreza, proteger el planeta y asegurar la prosperidad para todos como parte de una nueva agenda de los Objetivos de Desarrollo Sostenible (ODS). Cada uno de los ODS tiene metas específicas que deben alcanzarse en los próximos 15 años. Para alcanzar estas metas, todos deben contribuir: los gobiernos, el sector privado, y la sociedad civil (2).

Una de las metas de los ODS es la relacionada al acceso universal al agua potable, saneamiento e higiene, lo que no puede lograrse sin prestar la debida atención a las desigualdades en el acceso observadas entre diferentes grupos (ricos y pobres, poblaciones rurales y urbanas, o grupos desfavorecidos frente a la población general). Contar con datos desglosados y de mejor calidad sobre el acceso al agua, el saneamiento y la higiene permitiría

Tabla 1. Avances en el mundo sobre disponibilidad de agua potable, instalaciones de saneamiento y la disposición de excretas al aire libre

\begin{tabular}{|c|c|}
\hline Año 1990 & Año 2015 \\
\hline Población mundial: 5300 millones & Población mundial: 7300 millones \\
\hline El $57 \%$ de la población mundial vivía en zonas rurales & El 54\% de la población mundial vive en zonas urbanas \\
\hline $\begin{array}{l}\text { El } 76 \% \text { de la población utilizaba fuentes de agua potable } \\
\text { mejoradas }\end{array}$ & El 91\% de la población utiliza fuentes mejoradas de agua potable \\
\hline $\begin{array}{l}1300 \text { millones de personas carecían de fuentes de agua potable } \\
\text { mejoradas }\end{array}$ & $\begin{array}{l}663 \text { millones de personas carecen de fuentes mejoradas de agua } \\
\text { potable }\end{array}$ \\
\hline 346 millones de personas utilizaban aguas de superficie & 159 millones de personas utilizan aguas de superficie \\
\hline $\begin{array}{l}\text { El } 54 \% \text { de la población utilizaba instalaciones de saneamiento } \\
\text { mejoradas }\end{array}$ & $\begin{array}{l}\text { El } 68 \% \text { de la población utiliza instalaciones de saneamiento } \\
\text { mejoradas }\end{array}$ \\
\hline $\begin{array}{l}\text { Casi la mitad de la población mundial carecía de saneamiento } \\
\text { mejorado }\end{array}$ & Una de cada tres personas carece de saneamiento mejorado \\
\hline $\begin{array}{l}\text { Una de cada cuatro personas en todo el mundo practicaba la } \\
\text { defecación al aire libre (1 } 300 \text { millones) }\end{array}$ & $\begin{array}{l}\text { Una de cada ocho personas en todo el mundo practica la defecación } \\
\text { al aire libre (946 millones) }\end{array}$ \\
\hline
\end{tabular}

Fuente: Adaptado del Informe 2015 del Programa Conjunto de la Organización Mundial de la Salud (OMS)/Fondo de las Naciones Unidad para la Infancia (UNICEF) de Monitoreo sobre el acceso a agua potable y saneamiento ${ }^{(1)}$ 
detectar las desigualdades allí donde ocurren e intervenir de forma más directa ${ }^{(7)}$.

En el caso del Perú, según un reporte del Instituto Nacional de Estadística e Informática (INEI) del 2017, la pobreza extrema afectó al 3,8\% de la población del país, equivalente a 1 millón 205 mil personas, el 54,1\% de los pobres se localiza en el área urbana y el $45,9 \%$ en el área rural. Según la región natural, el $46,2 \%$ de pobres están en la sierra, el $36,9 \%$ en la costa y el $16,9 \%$ en la selva. Asimismo, se indica que el $80,4 \%$ de viviendas se provee de agua por red pública, ya sea dentro o fuera de la vivienda. En el área urbana, este servicio cubre $83,2 \%$; mientras que en el área rural al $71,1 \%$ de las viviendas. El $71,3 \%$ de las viviendas contaba con servicio higiénico conectado a red pública, 12,0 puntos porcentuales más que en el 2012. En el área urbana $87,2 \%$ de las viviendas contaba con este tipo de servicio higiénico, en cambio en el área rural solo el $20,0 \%$ disponía de este servicio ${ }^{(8)}$; todo lo cual determina la presencia de enfermedades asociadas a la carencia de estos servicios.

Bajo este escenario, el objetivo del presente artículo es mostrar las diferentes enfermedades que están relacionadas con el agua, así como también, describir el aumento de estas enfermedades con el cambio climático.

\section{GRUPOS DE ENFERMEDADES INFECCIOSAS RELACIONADAS CON EL AGUA}

Como se describió anteriormente es vital que la población tenga accesoal agua segura yal saneamiento básico, porque es conocido que la falta de estos servicios condiciona la presencia de diferentes tipos de enfermedades que afectan la salud de las poblaciones. Para una mejor visión de las enfermedades relacionadas con el agua, podemos ver en la
Tabla 2 una forma de agruparlas y explicar los mecanismos mediante los cuales se vehiculizan los agentes patógenos causantes de enfermedades e igualmente se ponen algunos ejemplos de estas infecciones. Si correlacionamos podremos notar que varias de las infecciones descritas están en el Perú y se presentan en forma de epidemias.

El estudio de Yang et al. muestra que a escala global, las enfermedades infecciosas asociadas con el agua están significativamente correlacionadas con factores socioambientales afectando a todas las regiones que se ven perjudicadas desproporcionadamente por diferentes categorías de enfermedades infecciosas ${ }^{(9)}$. Este mismo estudio muestra que entre 1991 y 2008, ocurrieron un total de 1428 brotes reportados y esto sólo representa «la punta del iceberg» del problema. Los brotes asociados a las enfermedades infecciosas están significativamente correlacionadas con factores sociales y ambientales. En la Figura 1 se puede mostrar la distribución de enfermedades a nivel global, según los ajustes hechos en la Tabla 2.

\section{ENFERMEDADES INFECCIOSAS PORTADAS POR EL AGUA}

Estas infecciones ocurren principalmente por la contaminación del agua con agentes infecciosos de modo que los humanos al consumirla serán afectados, entre ellas contamos a las enfermedades diarreicas agudas bacterianas como el cólera y otros enteropatógenos, infecciones virales como los enterovirus entre ellos la polio, hepatitis viral A y hepatitis viral $\mathrm{E}$, parasitosis intestinales, entre otros.

La afección más representativa de este grupo de enfermedades es la diarrea cuya etiología es variada. Según la Encuesta Demográfica y de Salud Familiar (ENDES) 2017 realizada por el INEI, en el Perú la prevalencia

Tabla 2. Clasificación de las enfermedades infecciosas relacionadas con el agua

\begin{tabular}{|c|c|c|}
\hline Clasificación & Mecanismo & Ejemplos \\
\hline $\begin{array}{l}\text { Portadas o transportadas por el } \\
\text { agua }\end{array}$ & Contaminación fecal & $\begin{array}{l}\text { Cólera, tifoidea, enteropatógenos, VHA, VHE, } \\
\text { enterovirus, parasitosis intestinal }\end{array}$ \\
\hline Soportados por el agua & $\begin{array}{l}\text { Organismos que parte de su ciclo de vida pasan } \\
\text { en el agua }\end{array}$ & Fasciolosis, paragonimiosis, leptospirosis. \\
\hline Vinculados con el agua & $\begin{array}{l}\text { Vectores biológicos que parte importante de su } \\
\text { ciclo de vida se da en el agua }\end{array}$ & $\begin{array}{l}\text { Malaria, dengue, zika, fiebre amarilla, } \\
\text { chikungunya }\end{array}$ \\
\hline Lavadas por el agua & $\begin{array}{l}\text { Relacionados a pobre higiene personal y al } \\
\text { contacto con agua contaminada }\end{array}$ & Pediculosis, ricketsiosis \\
\hline Dispersadas por el agua & $\begin{array}{l}\text { Organismos que proliferan en el agua y entran por } \\
\text { el tracto respiratorio }\end{array}$ & Legionelosis \\
\hline
\end{tabular}

VHA: virus de la hepatitis A; VHE: virus de la hepatitis $E$.

Fuente: Adaptado de Yang et al. (9). 


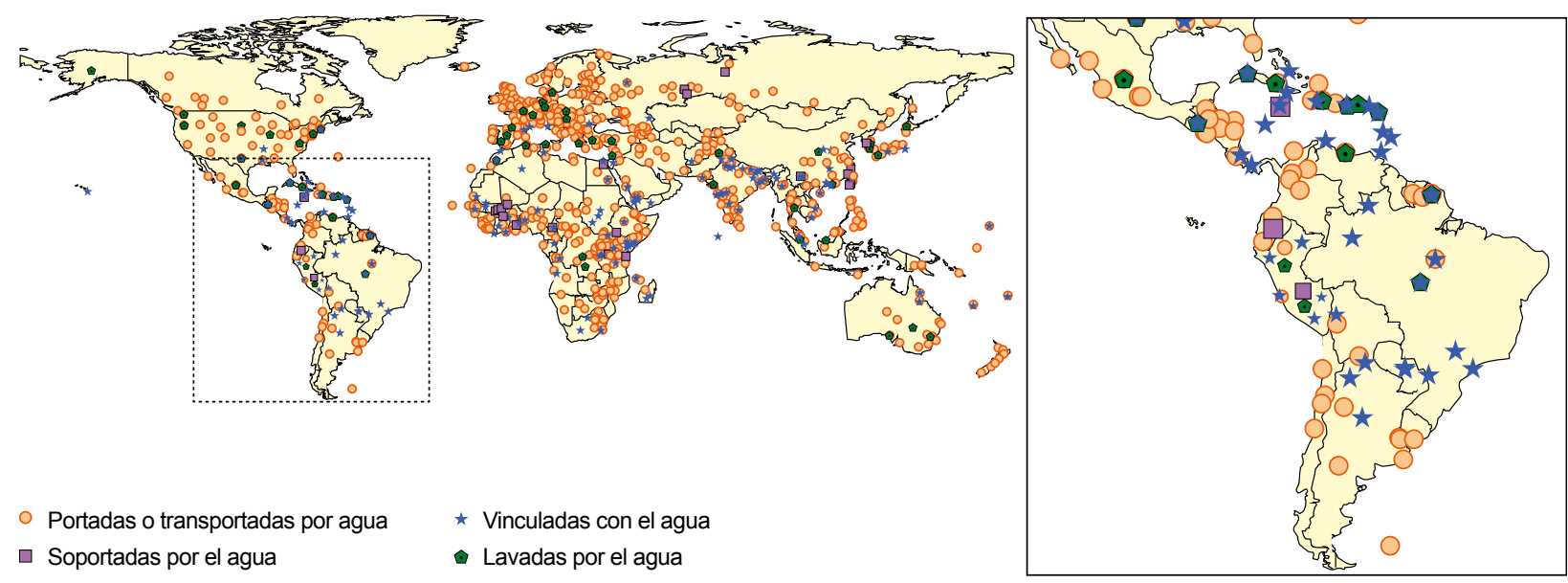

Figura 1. Distribución de brotes de enfermedades infecciosas relacionadas con el agua reportados de 1991 al 2008.

Fuente: Adaptado de Yang et al. (9).

de diarrea en niños menores de cinco años alcanzó $11,0 \%$, la cual es menor a la estimada para el $2012(12,3 \%)$, no habiéndose encontrado diferencias importantes por área de residencia $\left(10,6 \%\right.$ urbana y $12,2 \%$ rural) ${ }^{(8)}$.

Los patógenos más relevantes en la salud pública son los asociados con la mayor carga de morbilidad, gravedad, complicaciones y mortalidad. En nuestro país, el norovirus, Campylobacter y E. coli diarreogénico son los patógenos más prevalentes a nivel comunitario en los niños ${ }^{(10)}$. Por otro lado, hay estudios que muestran la presencia de enterovirus con manifestaciones neurológicas que tienen un comportamiento epidemiológico estacional con un claro aumento del número de casos en los meses de verano ${ }^{(11)}$. La epidemia con manifestaciones del síndrome de Guillain Barré que se presentó durante este año en Trujillo también está vinculado a enterovirus ${ }^{(12)}$.

Es importante recordar la epidemia del cólera que se inició en el Perú en enero de 1991 y se extendió a otros países de Sudamérica que conllevó a tener más de 533000 casos y 4700 defunciones en 19 países de este hemisferio ${ }^{(13)}$. Posteriormente, en el fenómeno de El Niño 1997-1998 se volvió a presentar el cólera en el norte del país, epidemia que tuvo proporcionalmente una mayor letalidad por que rápidamente se dejó de considerar la presunción diagnóstica y el manejo de casos ${ }^{(14)}$.

Una revisión realizada por Náquira et al. (15) de las enteroparasitosis a nivel nacional muestra la prevalencia de enteroparasitosis como Taenia sp y $S$. stercoralis en Tumbes, Diphilobotrium pacificum en Piura, $H$. nana en Ayacucho, A. lumbricoides y $T$. trichura en Loreto. En la costa prevalece la Taenia $s p, D$. pacificum y Enterovious vermicularis; en la selva alta: $A$. lumbricoides y S. stercolaris; en la selva baja: T. trichiura, uncinarias y E. vermicularis; y en la puna $H$. nana, es probable que estas condiciones no hayan tenido mucha variación en la medida que las mejoras del acceso al agua y saneamiento aún es limitado.

Por su parte, la hepatitis viral A en el Perú, tiene una prevalencia variable según el acceso a las condiciones de saneamiento, así en un estudio realizado en Lima, Arequipa, Iquitos, Piura y Cajamarca por Vildósola et al. (16) encuentran una prevalencia promedio de $50,5 \%$, siendo mayor en el nivel socioeconómico del grupo $\mathrm{E}$ con el $64,6 \%$. Igualmente se describe la presencia de antecedentes de infección por hepatitis $E$ en grupos de riesgo en el país (17). Por otro lado, en un estudio desarrollado por Cabada ${ }^{(18)}$, se encuentra una frecuencia del $24 \%$ de diarreas en turistas que visitaron Cusco, lo cual implica además del impacto sobre los visitantes, la necesidad de plantear una serie de precauciones y medidas de control.

\section{ENFERMEDADES INFECCIOSAS SOPORTADAS POR ELAGUA}

Comprende las enfermedades en las cuales una parte de su ciclo de transmisión se da en el agua, pero que requieren de intermediarios, un ejemplo de ello es el caso de la fasciolosis humana que tiene como intermediario al caracol. Esta enfermedad es endémica en la sierra y la costa, pero esporádica en la Amazonía, es reportada en 18 departamentos peruanos, mientras que la infección animal está presente en 21 de los 24 departamentos del Perú. La transmisión ocurre en las poblaciones rurales andinas dedicadas a la agricultura por ingestión de vegetales contaminados. El impacto negativo de la fasciolosis en la 
economía ganadera no es menor a USD 50 millones por año ${ }^{(19)}$, y considerando que se da en poblaciones pobres, el impacto es significativo para las familias.

Otra de las enfermedades que podemos catalogar en este grupo es la leptospirosis cuyo agente etiológico habitualmente es eliminado por reservorios que contaminan el agua, aunque últimamente se le ha encontrado viable en el medio ambiente ${ }^{(20)}$. Ocurre mundialmente, pero es endémica principalmente en países con climas húmedos subtropicales y tropicales. Estimaciones indican que a nivel mundial hay más de 500000 casos de leptospirosis anualmente. Es una enfermedad de potencial epidémico, principalmente después de lluvias fuertes o inundaciones. Se han registrado brotes en Brasil, Nicaragua, Guyana, Perú y en varios otros países de América Latina; aunque se han descrito casos en la mayoría de los países de las Américas. La mayoría de casos registrados tienen una manifestación severa, por lo cual mortalidad es mayor de $10 \%{ }^{(21)}$

En el Perú entre 2004 y 2017 el promedio anual de casos reportados está entre 476 y 2370 , siendo Loreto la región con mayor porcentaje de reportes (57\%); asimismo, se reporta en el $26 \%$ de pacientes febriles en el norte de Perú en población con pobres condiciones de saneamiento ${ }^{(22,23)}$.

\section{ENFERMEDADES INFECCIOSAS VINCULADAS AL $A G U A$}

En este grupo de enfermedades, los vectores que transmiten los virus, parásitos o bacterias cumplen una parte de su ciclo de vida en el agua. Una de ellas es la malaria que es transmitida por el vector del género Anopheles que está presente en la costa norte y la Amazonía, constituyendo un importante problema de salud pública en el Perú. Se han hecho esfuerzos para erradicarla desde la década de los 40 , por lo que, las autoridades sanitarias nacionales desarrollaron entre 1955 y 1970 diversas acciones para alcanzar este objetivo y proyectarse al control sostenible del daño durante la década de 1980. Si bien hubo una reducción importante de casos, se presentaron epidemias asociadas al fenómeno de El Niño en la costa norte.

En los últimos años, entre el 2013 y 2017 se han reportado anualmente entre 2509 y 3421 casos de malaria por Plasmodium vivax y en ese mismo periodo entre 410 y 1286 casos de malaria por Plasmodium falciparum, este último casi confinado a la región Loreto, mientras el Plasmodium vivax está presente además de Loreto en Amazonas, Junín, La Libertad, Ucayali, Cusco y Ayacucho ${ }^{(24)}$. Hasta el momento han sido incriminados como vectores principales de la transmisión de la malaria en el Perú al Anopheles darlingi, A. pseudopunctipennis, $A$. albimanus y $A$. benarrochi, y como secundarios las especies $A$. calderoni, $A$. nuneztovari, $A$. rangeli, $A$. oswaldoi, $A$. trinkae (= dunhami) y $A$. evansae, y como accidentales $A$. mattogrossensis y $A$. fluminensis, que por su dispersión en el país y los efectos del cambio climático, siempre constituirán un riesgo para la transmisión de la malaria ${ }^{(25)}$.

El dengue, el zika, el chikungunya (estas dos últimas de ingreso reciente al país) y la fiebre amarilla urbana, tienen como común denominador al vector Aedes aegypti que es un vector intradomiciliario y que está más vinculado a la disposición del agua, tanto en las zona lluviosas de la Amazonía y aún en las zonas donde no hay precipitaciones fluviales como en parte de la costa peruana. En la Amazonía la presencia de objetos inservibles que mantienen el agua de lluvia constituyen los principales criaderos de este mosquito. En las zonas donde no llueve y hay carencia de la red de agua potable las personas acumulan agua en el intra y peridomicilio, lo cual constituye los principales criaderos del Aedes aegypti, y en ambas zonas la administración intermitente del servicio de agua hace también que la población acumule agua en el intradomicilio y persista el problema de los criaderos.

Aproximadamente 2500 millones de personas en todo el mundo viven en áreas endémicas del dengue y están en riesgo de contraer la infección ${ }^{(26)}$. Cada año, se producen hasta 390 millones de infecciones por dengue, lo que resulta en un estimado de 96 millones de casos sintomáticos ${ }^{(27)}$. En las Américas, la incidencia del dengue ha aumentado continuamente desde la reintroducción de su vector, Aedes aegypti, en la década de $1970{ }^{(28)}$.

Al igual que en el Perú en varios países de las Américas una mayor incidencia de casos de dengue se asocia a deficientes condiciones sanitarias y también a un menor nivel educativo ${ }^{(29)}$, así en el Brasil, se observó que el bajo nivel socioeconómico de las comunidades se asocia de forma independiente con un mayor riesgo de dengue, lo que indica que dentro de las comunidades de tugurios con altos niveles de pobreza absoluta, los factores asociados con el gradiente social influyen en la transmisión del dengue ${ }^{(30)}$.

La gran dispersión del Aedes aegypti en el Perú, tanto en la costa como en la Amazonía y en particular la presencia de este vector en áreas de fiebre amarilla selvática, nos alerta del riesgo de reurbanización de esta enfermedad ${ }^{(31)}$, que sería crítico si no tenemos buenas coberturas o al menos la disponibilidad de la vacuna antiamarílica, por lo que es evidente que la medida más concreta para eliminar este vector es evitar la presencia de sus criaderos y eso se podría lograr con un abastecimiento adecuado de agua y de saneamiento.

La Figura 2 nos muestra la tendencia de los casos de dengue en el Perú entre 1990 y 2017. Asimismo, en 


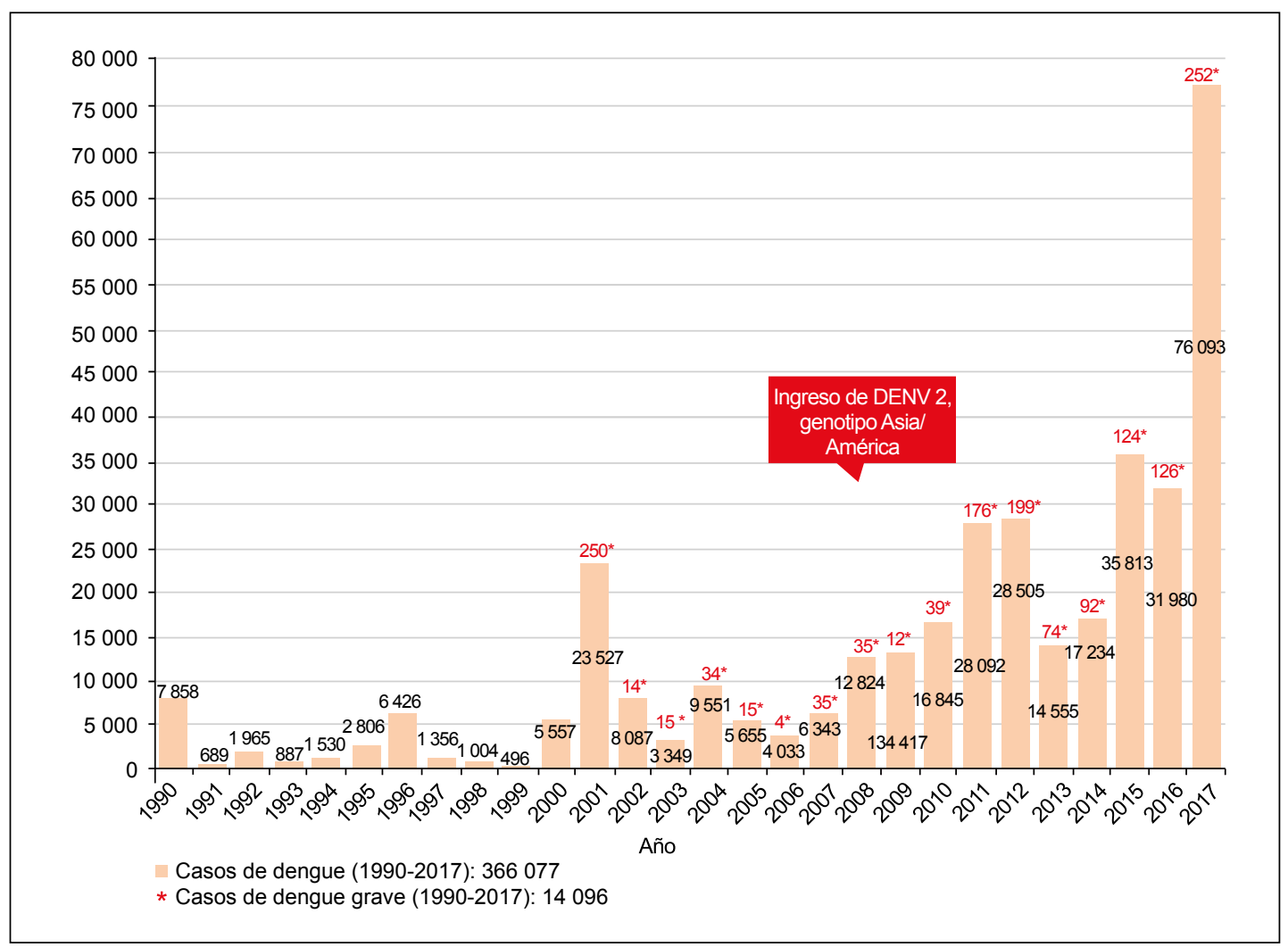

Figura 2. Tendencia histórica de los casos de dengue en el Perú desde su ingreso en 1990 al 2017

Fuente: Centro Nacional de Epidemiología y Control de Enfermedades - Ministerio de Salud.

la Figura 3 se puede apreciar la dispersión del Aedes aegypti en el territorio peruano.

Un factor determinante que se agrega al problema del agua en el mundo y en nuestro país es el cambio climático y los fenómenos naturales cada vez más frecuentes, por lo que el cambio climático debe considerarse fundamentalmente como un problema de justicia global. Comprender la compleja interacción de las tendencias climáticas y socioeconómicas es imprescindible para proteger la salud humana y disminuir la carga de enfermedades como el dengue. Se espera que la temperatura, la lluvia y la frecuencia de los desastres naturales, así como las tendencias no climáticas relacionadas con el crecimiento y la migración de la población, la urbanización y el comercio y los viajes internacionales aumenten la prevalencia de criaderos de mosquitos, la supervivencia del mosquito y la velocidad de reproducción del mosquito, la velocidad de incubación viral, la distribución del virus del dengue y sus vectores, los patrones de migración humana hacia áreas urbanas y el desplazamiento después de desastres naturales ${ }^{(32-34)}$.

\section{ENFERMEDADES INFECCIOSAS LAVADAS POR EL AGUA}

Hay enfermedades asociadas a la falta de higiene y que se derivan de la carencia de agua que exacerba las precarias

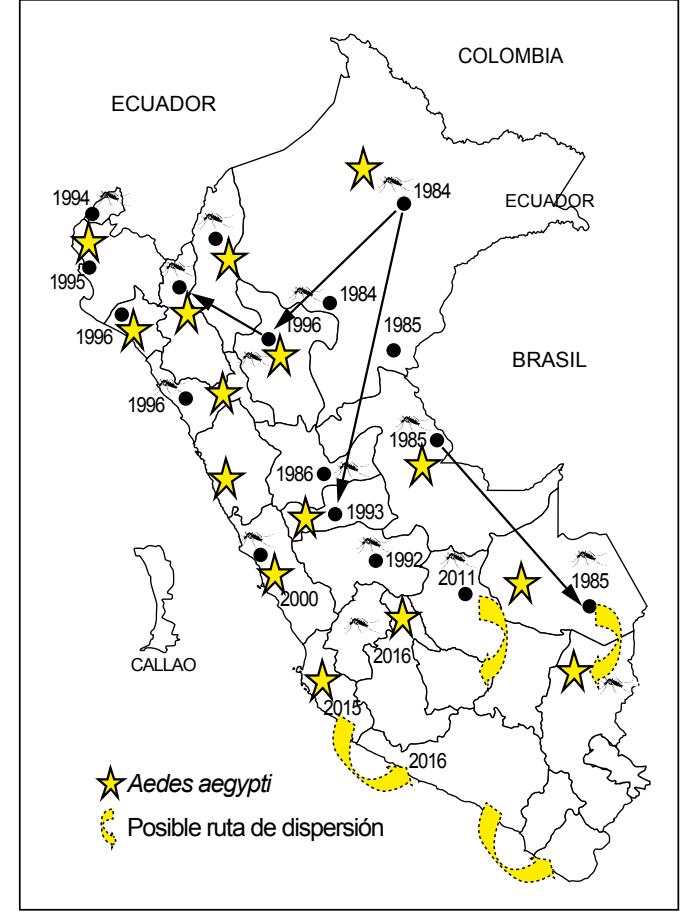

Figura 3. Dispersión del Aedes aegypti al 2018.

Fuente: Elaboración propia en base a información del Instituto Nacional de Salud. 
condiciones de vida de comunidades sobre todo rurales, como es el caso de las pediculosis, acarosis, y rickettsiosis que están presente en comunidades desfavorecidas del mundo y de nuestro país ${ }^{(35-38)}$.

\section{ENFERMEDADES INFECCIOSAS DISPERSADAS POR ELAGUA}

Una de las enfermedades que son dispersadas por el agua es la legionelosis, un tipo de bacteria que se encuentran naturalmente en el agua dulce. Cuando las personas se exponen a estas bacterias, pueden enfermarse (enfermedad del legionario y fiebre de Pontiac). Estas bacterias crecen mejor en agua caliente, como la que se encuentra en bañeras de hidromasaje o jacuzzis, torres de refrigeración (unidades de aire acondicionado para edificios grandes), tanques de agua caliente, y sistemas de plomería grandes. La enfermedad muestra una distribución mundial con representación en América del Norte, Sudamérica, Asia, África, Australia y Europa ${ }^{(39-41)}$.

\section{CONCLUSIÓN}

La carencia de agua segura y de un adecuado saneamiento, últimamente asociado al cambio climático y los desastres naturales condicionan la permanencia de enfermedades relacionadas al agua, por lo que la estrategia para minimizar estos efectos es el trabajo multisectorial, teniendo como una de las referencias los Objetivos de Desarrollo Sostenibles. El objetivo relacionado al acceso universal a agua potable, saneamiento e higiene no podrá lograrse si no se reducen las inequidades en el acceso al agua observadas entre diferentes grupos con diferentes condiciones económicas, poblaciones rurales y urbanas, o grupos desfavorecidos frente a la población general.

\section{Fuentes de financiamiento: autofinanciado.}

Declaración de conflictos de interés: el autor no refiere conflictos de interés.

\section{REFERENCIAS BIBLIOGRÁFICAS}

1. Organización Mundial de la Salud. Informe 2015 del PCM sobre el acceso a agua potable y saneamiento: datos esenciales [Internet]. Geneva: OMS; c2018 [citado el 11 de mayo de 2018]. Disponible en: http:// www.who.int/water_sanitation_health/ monitoring/jmp-2015-key-facts/es/

2. World Health Organization; United Nations Children's Fund (UNICEF). Progress on drinking water, sanitation and hygiene [Internet]. Geneva: WHO; 2017 [citado el 11 de mayo de 2018]. Disponible en: http:// apps.who.int/iris/bitstream/hand le/10665/258617/9789241512893eng.pdf

3. Levy K, Smith SM, Carlton EJ. Climate change impacts on waterborne diseases: moving toward designing interventions. Curr Environ Health Rep. 2018;5(2):272-82.

4. Booth M. Climate change and the neglected tropical diseases. Adv Parasitol. 2018;100:39-126.

5. Chretien JP, Anyamba A, Small J, Britch S, Sanchez JL, Halbach AC, et al. Global climate anomalies and potential infectious disease risks: 2014-2015. PLoS Curr. 2015 Jan 26;7. pii: ecurrents. outbreaks.95fbc4a8fb4695e049baabfc$2 \mathrm{fc} 8289 \mathrm{f}$.

6. Suarez-Ognio Luis, Estela-Ayamamani David, Cáceres-Mejía Brenda, Gambirazio-Carbajal Carlos, Cabrera Rufino. Impacto del fenómeno «El Niño» de 1997-1998 en la salud de la población peruana, riesgo potencial para el 2015.
Rev Peru Med Exp Salud Publica. 2015;32(2):403-4.

7. Naciones Unidas. Objetivos de Desarrollo Sostenible [Internet]. New York: Naciones Unidas; 2016 [citado el 11 de mayo de 2018]. Disponible en: https:// www.un.org/sustainabledevelopment/es/ water-and-sanitation/

8. Instituto Nacional de Estadística e Informática. Evolución de la pobreza monetaria 2007-2017. Lima: INEI; 2017 [citado el 11 de mayo de 2018]. Disponible en: https://www.inei.gob.pe/media/ cifras_de_pobreza/pobreza2016.pdf

9. Yang K, Lejeune J, Alsdorf D, Lu B, Shum CK, Liang S. Global distribution of outbreaks of water-associated infectious diseases. PLoS Negl Trop Dis. 2012;6(2):e1483.

10. Riveros M, Ochoa TJ. Relevant public health enteropathogens. Rev Peru Med Exp Salud Publica. 2015;32(1):157-64.

11. Espinoza Iván $\mathrm{O}$, Ochoa Theresa J, Mosquito S, Barletta F, Hernández R, Medina MP, et al. Infecciones del sistema nervioso central por enterovirus en niños atendidos en un hospital de Lima, Perú. Rev Peru Med Exp Salud Publica. 2011;28(4):602-9.

12. Ministerio de Salud del Perú. Síndrome de Guillain Barre,- Perú al 14 Junio (SE 24) 2018 [Internet]. Lima: MINSA; [citado el 11 de mayo de 2018]. Disponible en: http://www.dge.gob.pe/portal/docs/ tools/sg/SSD140618.pdf
13. Swerdlow DL, Mintz ED, Rodriguez M, Tejada E, Waterborne transmission of epidemic cholera in Trujillo, Peru: lessons for a continent at risk. Lancet. 1992;340(8810):28-33.

14. Organización Panamericana de la Salud; Organización Mundial de la Salud. Cambio climático y enfermedades infecciosas: consecuencias del fenómeno el niño [Internet]. Washington, D.C.: PAHO; 1998 [citado el 11 de mayo de 2018]. Disponible en: http://www.bvsde.paho.org/acrobat/ninofen.pdf

15. Oficina General de Epidemiología, Ministerio de Salud del Perú. Helmintos intestinales en el Perú, análisis de la prevalencia (1981-2001) [Internet]. Lima: MINSA; [citado el 11 de mayo de 2018]. Disponible en: http://bvs.minsa. gob.pe/local/OGE/235_OGE31.pdf

16. Hernández Roger, Chaparro Eduardo, Díaz Carlos, Carbajal Martha, Cieza Érico, Cerpa Rosario. Frecuencia de hepatitis a en niños y adolescentes de cinco ciudades del Perú. Rev Peru Med. Exp Salud Publica. 2015;32(3):499-503.

17. Vildósola H, Colichón A, Barreda M, Piscoya J, Palacios O. Seroprevalencia de anticuerpos $\operatorname{IgG}$ contra el vírus de la hepatitis $\mathrm{E}$ en un grupo de riesgo en Lima. Rev Gastroenterol Peru. 2000;20(2):111-6.

18. Cabada MM, Maldonado F, Quispe W, Mozo K, Serrano E, González E, et al. Risk factors associated with diarrhea among international visitors to Cuzco, Peru. Am J Trop Med Hyg. 2006;75(5):968-72. 
19. Espinoza JR, Terashima A, Herrera-Velit P, Marcos LA. Human and animal fascioliasis in Peru: impact in the economy of endemic zones. Rev Peru Med Exp Salud Publica. 2010;27(4):604-12.

20. Casanovas-Massana A, Pedra GG, Wunder EA Jr, Diggle PJ, Begon M, Ko AI. Quantification of Leptospira interrogans Survival in Soil and Water Microcosms. Appl Environ Microbiol. 2018;84(13):e00507-18.

21. Organización Panamericana de la Salud; Organización Mundial de la Salud. Leptospirosis [Internet]. Washington, D.C.: PAHO; 1998 [citado el 11 de mayo de 2018]. Disponible en: https://www.paho. org/hq/index.php?option=com_conten$\mathrm{t} \&$ view $=$ article\&id $=7821 \% 3 \mathrm{~A} 2012$-informacion-general-leptospirosis\&cati$\mathrm{d}=4784 \% 3$ Aleptospirosis-contents\&Ite$\mathrm{mid}=0$ \&lang $=\mathrm{es} 6 \%$

22. Silva-Díaz H, Llatas-Cancino DN, Campos-Sánchez MJ, Aguilar-Gamboa FR, Mera-Villasis KM, Valderrama-Ayén MY. Leptospirosis frequency and sociodemographic characteristics associated in febrile patients from northern Perú. Rev Chilena Infectol. 2015;32(5):530-5.

23. Ministerio de Salud del Perú. Situación Epidemiológica de la Leptospirosis en el Perú [Internet]. Lima: MINSA; 2017 [citado el 11 de mayo de 2018]. Disponible en: http://www.dge.gob.pe/portal/docs/ tools/teleconferencia/SE112017/01.pdf

24. Ministerio de Salud del Perú. Número de casos de malaria. Perú 2013 - 2018 [Internet]. Lima: MINSA; 2017 [citado el 11 de mayo de 2018]. Disponible en: http://www.dge.gob.pe/portal/docs/ vigilancia/sala/2018/SE05/malaria.pdf

25. Instituto Nacional de Salud, Ministerio de Salud. Distribución de los principales insectos vectores de enfermedades en el Perú. Lima: INS; 2002.

26. World Health Organization. Dengue and severe dengue [Internet]. Geneva: WHO; 2014 [citado el 11 de mayo de 2018].
Disponible en: http://www.who.int/ mediacentre/factsheets/fs117/en/

27. Bhatt $S$, Gething PW, Brady OJ, Messina JP, Farlow AW, Moyes CL, et al. The global distribution and burden of dengue. Nature. 2013;496(7446):504-7.

28. Guzman MG, Halstead SB, Artsob H, Buchy P, Farrar J, Gubler DJ, et al. Dengue: a continuing global threat. Nat Rev Microbiol. 2010;8(12 Suppl):S7-16.

29. Organización Panamericana de la Salud; Organización Mundial de la Salud. Últimos adelantos técnicos en la prevención y el control del dengue en la Región de las Américas. Informe de reunión (28-29 de mayo del 2014, Washington,DC) [Internet]. Washington, DC: PAHO; 2014 [citado el 11 de mayo de 2018]. Disponible en: http://iris.paho. org/xmlui/handle/123456789/31294

30 Kikuti M, Cunha GM, Paploski IAD, Kasper AM, Silva MMO, Tavares AS, et al. Spatial distribution of dengue in a brazilian urban slum setting: role of socioeconomic gradient in disease risk. PLoS Negl Trop Dis. 2015;9(7):e0003937.

31. Codeço CT, Luz PM, Struchiner CJ. Risk assessment of yellow fever urbanization in Rio de Janeiro, Brazil. Trans R Soc Trop Med Hyg. 2004;98(12):702-10.

32. Chang AY, Fuller DO, Carrasquillo O, Beier JC. Social justice, climate change, and dengue. Health Hum Rights. 2014;16(1):93-104.

33. Campbell LP, Luther C, Moo-Llanes D, Ramsey JM, Danis-Lozano R, Peterson AT. Climate change influences on global distributions of dengue and chikungunya virus vectors. Philos Trans R Soc Lond B Biol Sci. 2015;370(1665):20140135.

34. Van Kleef E, Bambrick H, Hales S. The geographic distribution of dengue fever and the potential influence of global climate change. TropIKA.net [Internet]. 2010 [citado el 11 de mayo de 2018];2(1). Disponible en: http://journal.tropika. net/scielo.php?script $=$ sci_arttext\&pi$\mathrm{d}=$ S2078-86062010005000001
35. Arnaud A, Chosidow O, Détrez MA,Bitar D, Huber F, Foulet F, et al. Prevalences of scabies and pediculosis corporis among homeless people in the Paris region: results from two randomized crosssectional surveys (HYTPEAC study). $\mathrm{Br}$ J Dermatol. 2016;174(1):104-12.

36. Riabi HR, Atarodi A. Epidemiological and Clinical Study of Infested Cases with Pediculus capitis and P. corporis in Khorasan-e-Razavi, Iran.Iran J Parasitol. 2012;7(1):85-91.

37. Gutierrez EL, Galarza C, Ramos W, Tello M.Skin diseases in the Peruvian Amazonia. Int J Dermatol. 2010;49(7):794-800.

38. Forshey BM, Stewart A, Morrison AC, Gálvez H, Rocha C, Astete H, et al. Epidemiology of spotted fever group and typhus group rickettsial infection in the Amazon basin of Peru. Am J Trop Med Hyg. 2010;82(4):683-90.

39. Organización Mundial de la Salud. Legionelosis [Internet]. Geneva: OMS; 2018 [citado el 11 de mayo de 2018]. Disponible en: http://www.who.int/ es/news-room/fact-sheets/detail/ legionellosis

40. Silk BJ, Moore MR, Bergtholdt M, Gorwitz RJ, Kozak NA, Tha MM, et al. Eight years of Legionnaires' disease transmission in travellers to a condominium complex in Las Vegas, Nevada. Epidemiol Infect. 2012;140(11):1993-2002.

41. Blatny JM, Fossum H, Ho J, Tutkun M, Skogan G, Andreassen O, et al. Dispersion of Legionella-containing aerosols from a biological treatment plant, Norway. Front Biosci (Elite Ed). 2011;1(3):1300-9.

Correspondencia: César Cabezas Sánchez Dirección: Cápac Yupanqui 1400, Jesús María. Lima, Perú.

Correo electrónico: ccabezas@ins.gob.pe 